\title{
Multiple cerebral infarction associated with neuroleptic-induced lupus anticoagulant
}

\author{
J.D.E. Laugharne \\ University Department of Psychiatry, Northern General Hospital, Herries Road, Sheffield \\ S5 7AU, UK
}

\begin{abstract}
A 39 year old schizophrenic woman with a 20 year history of neuroleptic treatment suffered bilateral cerebral infarcts and coagulation studies revealed a lupus anticoagulant (LA). She highlights the poorly recognized risk of thrombosis in patients on neuroleptics who develop LA. In this case the LA may have been induced by long-term therapy with haloperidol, a novel and potentially important association.
\end{abstract}

Keywords: Cerebral infarction - Lupus anticoagulant - Neuroleptics - Haloperidol

\section{INTRODUCTION}

The relatively high incidence of immunological abnormalities induced by phenothiazines, especially chlorpromazine, is not an area with which many psychiatrists are familiar. However, the fact that such abnormalities do occur has been well documented (Zarrabi et al., 1979; Canosos and Sise, 1982) and of particular interest is the increased occurrence of the lupus anticoagulant (LA) in patients on phenothiazines. The LA is an antiphospholipid antibody, usually an IgG or IgM, which prolongs in vitro measurement of the partial thromboplastin time (PTT) but causes a hypercoagulable state in vivo (Boxer et al., 1976; Shapiro et al., 1981), thereby increasing the risk of thrombotic episodes. The patient described here had a history of treatment with various neuroleptics and sustained multiple cerebral infarcts, in association with a circulating lupus anticoagulant.

\section{CASE REPORT}

The patient, a 39 year old woman, was first diagnosed as schizophrenic at the age of 19 years. Following this diagnosis she was treated with various antipsychotic agents on an intermittent basis, including trifluoperazine, thioridazine and flupenthixol depot injections. She received chlorpromazine between 1980 and 1983 , at an average dose of $400 \mathrm{mg} /$ day but has not been prescribed this drug since then. From 1985 onwards she was maintained on haloperidol, initially in oral form, and from 1987 as a depot at $300 \mathrm{mg}$ monthly. There is no history of any other drugs previously associated with LA.

In December 1991 the patient, residing at the time on a psychiatric rehabilitation unit, developed rightsided limb and facial weakness, from which she made a rapid spontaneous recovery. One month later she experienced sudden onset facial weakness, more marked on the left than the right side, with accompanying dysphagia and dysarthria. Examination revealed signs of a pseudobulbar palsy, mild weakness of the right lower limb with a positive Babinski sign on the right side and no sensory deficit. There was no splenomegaly or adenopathy. An urgent magnetic resonance imaging (MRI) scan showed the presence of bilateral parietal infarcts (Fig. 1).

Clinical recovery from this episode was slow but steady until 2 months later when, whilst being investigated for the cause of these infarcts, she developed a further episode of left-sided hemiparesis, with a worsening of her dysarthria and dysphagia which has improved only minimally.

A clotting screen gave a prothrombin time (PT) of $14.1 \mathrm{~s} \mathrm{(control} \mathrm{time:} 10.5-14.0 \mathrm{~s}$ ) and a PTT of $51.5 \mathrm{~s}$ (control: 28-39 s), which was suggestive of the presence of LA. A dilute Russell's viper venom time (DRVVT) ratio of 1.9 (normal value 0.9-1.09) with a correction to 1.34 on addition of frozen-thawed platelets strongly supported the diagnosis (Creagh and Greaves, 1991). Anticardiolipin antibodies were normal. Antinuclear factor (ANF) was positive with a titre of 20, but all other autoantibodies tested for, including dsDNA, n-RNP, SM, SCL70, Ro and La, were negative. Erythrocyte sedimentation rate (ESR) was raised at $87 \mathrm{~mm} / \mathrm{h}$ but serum urea and electrolytes, glucose and liver function tests were unremarkable as were serum cholesterol and triglycerides. Total 


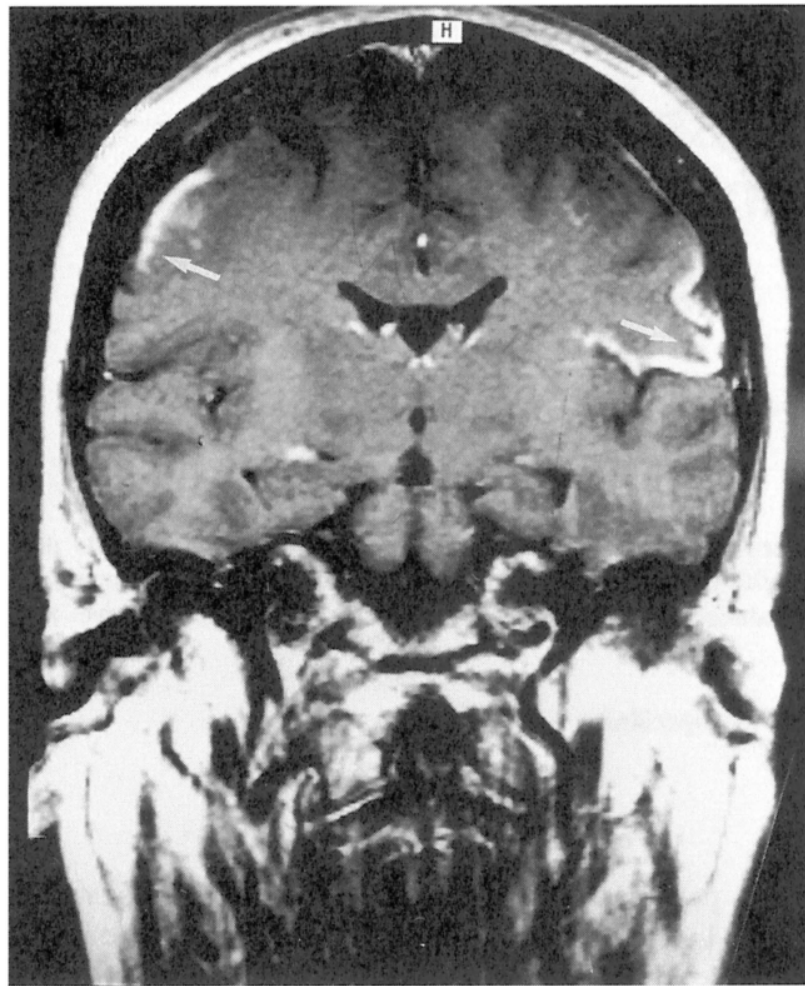

FIG. 1. Single coronal T1 weighted image from gadoliniumDTPA enhanced MRI shows gyral swelling bilaterally and adjacent gyral enhancement (arrowed) consistent with luxury perfusion in subacute infarctions.

serum protein and $\mathrm{IgG}$ and $\mathrm{IgM}$ fractions were within normal limits. Syphilis serology was negative. ECG and cardiac echogram were normal and Doppler studies showed evidence of minimal bilateral carotid artery disease, with less than $20 \%$ loss of lumen diameter and with good vertebral artery flow.

Neuroleptics were discontinued following the first ischaemic episode and have not been administered since. Following the third episode, the patient was commenced on aspirin $300 \mathrm{mg}$ daily and has suffered no further such insult to date.

\section{DISCUSSION}

LA was first described in patients with systemic lupus erythematosus (SLE) (Conley and Hartmann, 1952) where it is found in around 10\% of cases (Canosos and Sise, 1982), and also occurs in a range of disorders, including neoplasms, rheumatoid arthritis, autoimmune haemolytic anaemia and in women with a history of recurrent spontaneous abortions as well as in healthy individuals (El-Mallakh et al., 1988). It has also been reported to be induced by a variety of drugs, including procainamide, quinidine, phenytoin, valproic acid and phenothiazines (El-Mallakh et al., 1988).

Several workers have demonstrated immunological changes in patients on phenothiazines. Canosos and Sise (1982) reported a group of 42 patients treated with chlorpromazine (CPZ) or another phenothiazine but with a history of CPZ use. Thirty-eight per cent of these patients had LA, 33\% had antinuclear antibodies and $46 \%$ had elevated levels of serum IgM, whereas 53 controls showed no such abnormalities. Zarrabi et al. (1979) described a group of schizophrenic patients, in whom $75 \%$ of those who had received CPZ for longer than 2.5 years or were currently on a different phenothiazine but had previously taken CPZ for more than 2.5 years, had developed raised levels of $\operatorname{IgM}$ and circulating inhibitors of coagulation. Fifty per cent of their patients who had LA also had splenomegaly (which was not found in this patient).

LA is known to predispose to thrombotic events. Triplett et al. (1988) reported a thrombotic event in one of four patients with LA attributed to CPZ treatment and observed an increased risk of thrombosis in patients with drug-induced LA, compared with those with non-drug-induced LA. El-Mallakh et al. (1988) described a 35 year old woman who suffered multiple lucunar cerebral infarctions secondary to a CPZ-associated LA. In a retrospective study to determine the frequency of thrombosis in patients with the LA Mueh et al. (1980) described eight patients with phenothiazine-induced LA, three of whom had a history of a thrombotic event of which one was a cerebral infarct. Although El-Mallakh et al. (1988) suggest that thrombotic events are apparently a rarity in phenothiazine-induced LA, the literature is scanty and Triplett et al. (1988) and Mueh et al. (1980) suggest otherwise.

On the current available evidence it would appear that LA is induced by phenothiazines and not by other groups of neuroleptics (Zarrabi et al., 1979) and that CPZ is by far the most commonly implicated agent. Canosos and Sise (1982) comment that the presence of LA was observed as early as 3 months after starting CPZ therapy, but that the autoantibodies were more frequent in patients treated over a longer period. Although most patients with LA in their study had been exposed to a cumulative dose of CPZ greater than $1000 \mathrm{mg}$, LA development was observed with a minimum dose of $48 \mathrm{mg}$ indicating that in some patients relatively low exposure to $\mathrm{CPZ}$ can be immunogenic. They also noted that the persistence of immunological abnormalities, long after discontinuation of $\mathrm{CPZ}$ in patients maintained on other 
phenothiazines, suggested a cross-reaction between these compounds which perpetuated the antigenicity induced by the CPZ.

The patient reported here is of particular interest in that, although she had received chlorpromazine over a 3 year period in the past, her treatment during the 7 years prior to the first infarct was with haloperidol alone. The only previous case in the literature of haloperidol possibly being implicated as a cause of LA was one of the eight patients described by Mueh et al. (1980), who was apparently treated concurrently with haloperidol and thioridazine prior to an LArelated thrombotic event. The importance of haloperidol in this case has been called into doubt (El-Mallakh et al., 1988). The long period of time which had elapsed between my patient last receiving chlorpromazine and her first ischaemic event strongly raises the possibility that haloperidol and presumably other agents of the butyrophenone group may be capable of inducing LA. An alternative explanation is that haloperidol is capable, like non-CPZ phenothiazines, of perpetuating the antigenicity induced by $\mathrm{CPZ}$, but this seems unlikely in view of the marked difference in the molecular structure of these drugs.

In regard to the treatment of neuroleptic-induced LA, no clear management strategy or sense of longterm outcome has yet emerged (Kaslow et al., 1992). Discontinuation of the neuroleptic and commencement of anticoagulant therapy (heparin, warfarin or aspirin) have proved successful in patients with thrombotic complications, as with this patient. In this particular case aspirin was prescribed rather than warfarin due to difficulty ensuring the patient's cooperation with regular venepuncture for clotting function evaluation.

In conclusion, clinicians should be aware that phenothiazines, particularly CPZ, commonly induce immunological abnormalities including LA which can result in thrombotic events including infarction, the rarity of which is open to question.
This case also raises the possibility that other neuroleptics, in particular haloperidol, may also be capable of inducing LA.

\section{Acknowledgements}

The author wishes to thank Dr M. Peet, Mr P. Pratt, Dr M. Greaves and Dr A. Mason for their valuable comments and Mrs B. Nesbitt for secretarial assistance.

\section{REFERENCES}

Boxer M, Ellman L and Carvalho A (1976) The lupus anticoagulant. Arthritis and Rheumatism, 19, 1244-1248.

Canosos R and Sise H (1982) Chlorpromazine-induced lupus anticoagulant and associated immunologic abnormalities. American Journal of Haematology, 13, 121-129.

Conley CL and Hartmann RC (1952) A hemorrhagic disorder caused by circulating anticoagulants in patients with disseminated lupus erythematosus. Journal of Clinical Investigations, 31, 621-622.

Creagh MD and Greaves M (1991) Lupus anticoagulant. Blood Reviews, 5, 162-167.

El-Mallakh R, Donaldson J, Kranzler $\mathbf{H}$ and Raly A (1988) Phenothiazine-associated lupus anticoagulant and thrombotic disease. Psychosomatics, 29, 109-122.

Kaslow KA, Rosse RB, Zeller JA, Nagy MS and Deutsch SI (1992) Journal of Clinical Psychiatry, 53, 103-104 (letter).

Mueh JR, Herbst KD and Rapaport SI (1980) Thrombosis in patients with the lupus anticoagulant. Annals of Internal Medicine, 92, 156-159.

Shapiro SS, Thiagarajan P and DeMarco L (1981) Mechanism of action of the lupus anticoagulant. Annals of the New York Academy of Sciences, 370, 359-365.

Triplett DA, Brandt JT, Musgrave KA and Orr CA (1988) The relationship between lupus anticoagulants and antibodies to phospholipid. Journal of the American Medical Association, 259, 550-554.

Zarrabi M, Zucker S and Miller F (1979) Immunologic and coagulation disorders in chlorpromazine-treated patients. Annals of Internal Medicine, 991, 194-199.

(Received 15 March 1994; accepted as revised 26 August 1994) 


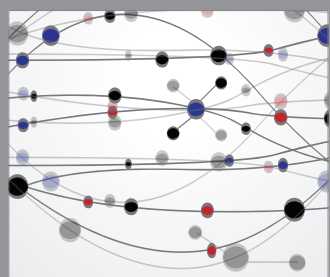

The Scientific World Journal
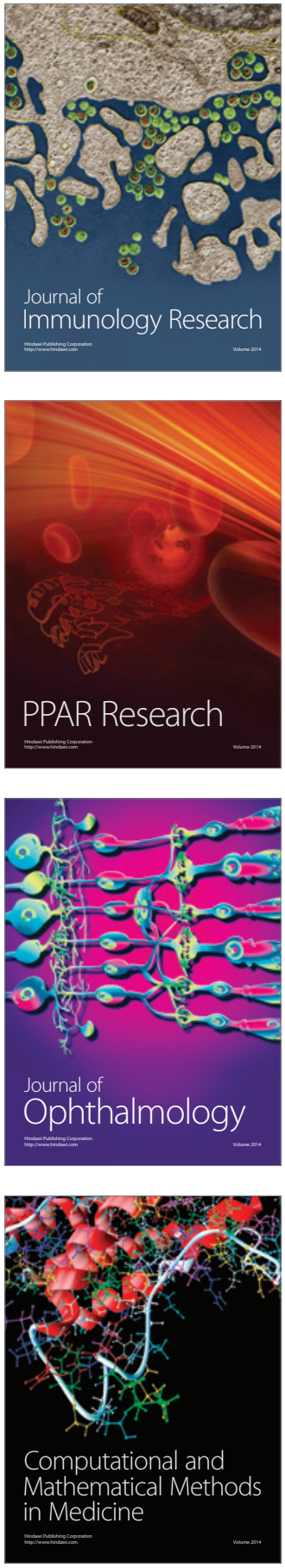

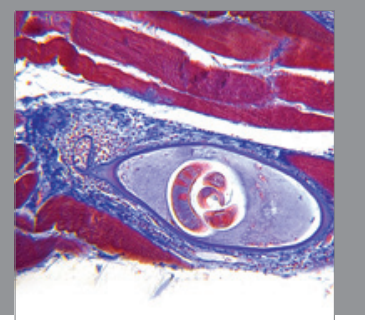

Gastroenterology

Research and Practice
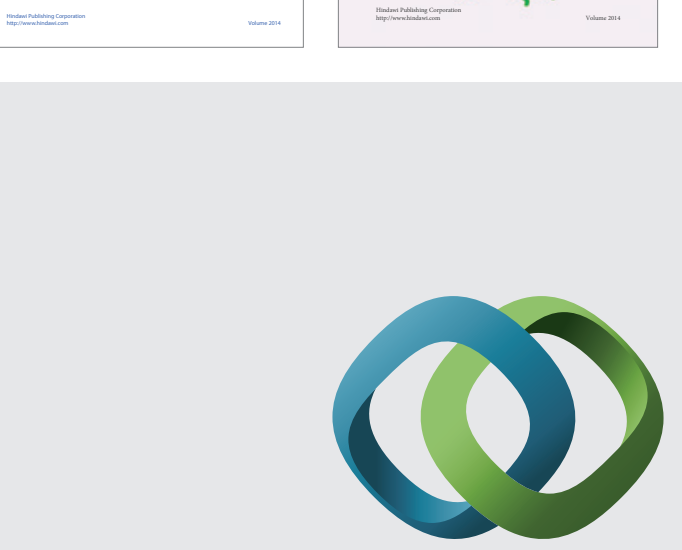

\section{Hindawi}

Submit your manuscripts at

http://www.hindawi.com
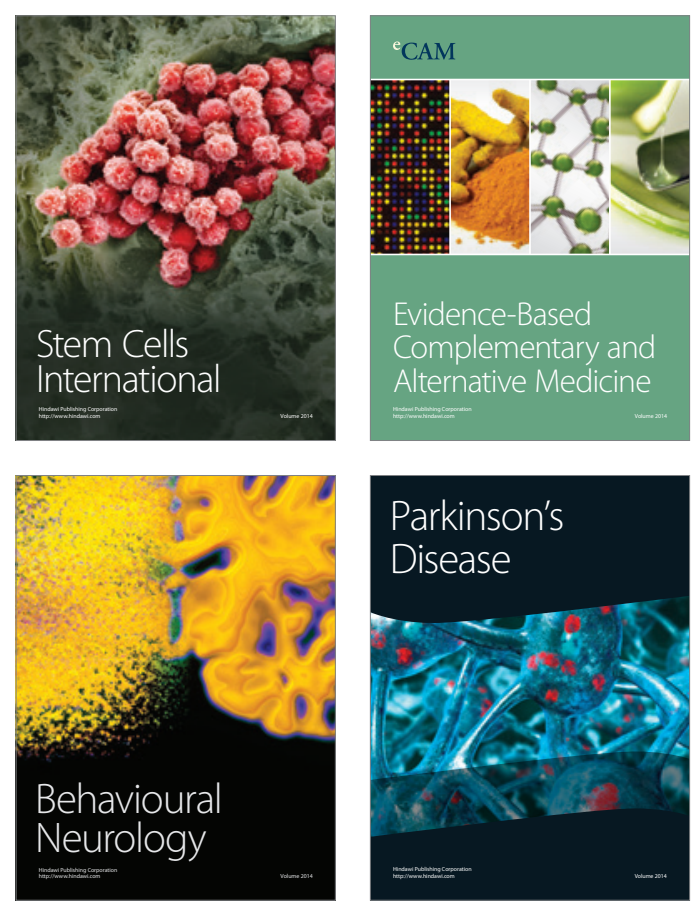

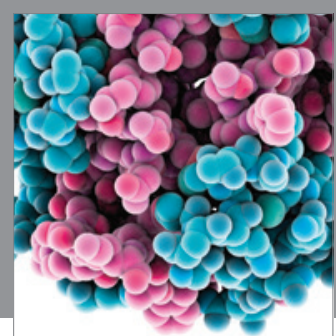

Journal of
Diabetes Research

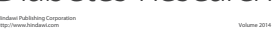

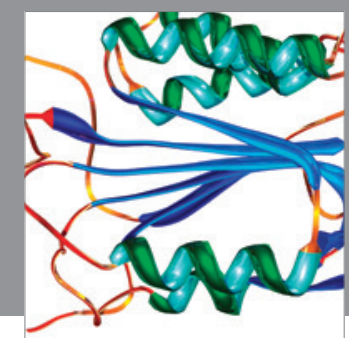

Disease Markers
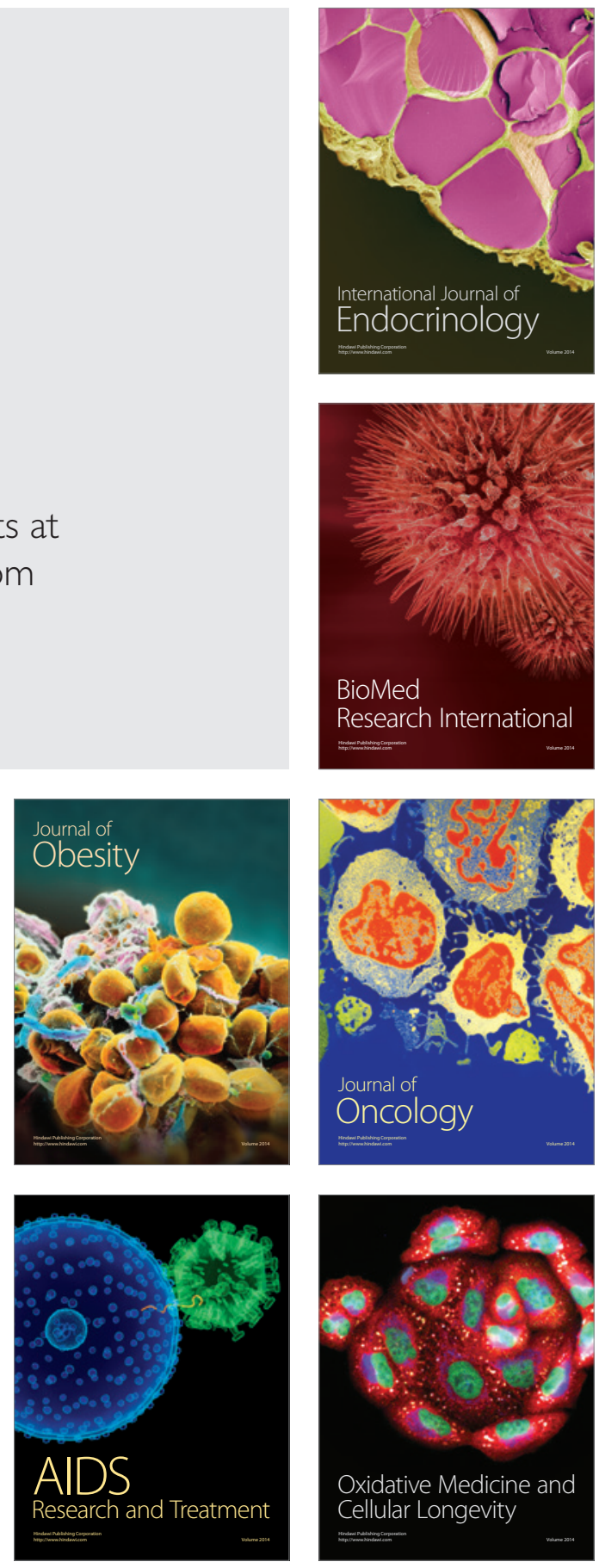